\title{
Le système éducatif en Géorgie
}

\section{Lisa Bydanova et Lela Maisuradze}

\section{(2) OpenEdition}

\section{Journals}

Édition électronique

URL : http://journals.openedition.org/ries/1006

DOI : 10.4000/ries. 1006

ISSN : 2261-4265

\section{Éditeur}

Centre international d'études pédagogiques

\section{Édition imprimée}

Date de publication : 1 septembre 2010

Pagination : 165-171

ISBN : 978-2-85420-582-4

ISSN : 1254-4590

\section{Référence électronique}

Lisa Bydanova et Lela Maisuradze, "Le système éducatif en Géorgie », Revue internationale d'éducation de Sèvres [En ligne], 54 I septembre 2010, mis en ligne le 01 septembre 2013, consulté le 21 décembre 2020. URL : http://journals.openedition.org/ries/1006 ; DOI : https://doi.org/10.4000/ries.1006

Ce document a été généré automatiquement le 21 décembre 2020.

(c) Tous droits réservés 


\title{
Le système éducatif en Géorgie
}

\author{
Lisa Bydanova et Lela Maisuradze
}

1 Jusqu'au début des années quatre-vingt-dix, la Géorgie ${ }^{1}$ faisait partie des quinze républiques de l'URSS. En avril 1991, elle déclare son indépendance et devient la République démocratique de Géorgie. Cette période est également marquée par d'importantes réformes économiques et sociales mises en place suite au passage de l'économie planifiée à l'économie de marché libre. En 2004, par la Révolution des roses, un gouvernement pro-occidental mené par un jeune leader politique, Mikhail Saakashvili, accède au pouvoir. Le nouveau président se donne comme objectif d'accélérer la transition économique et de se rapprocher du monde occidental. Un accent particulier est mis sur la lutte contre la corruption et la rénovation du secteur public. Parmi les secteurs les plus concernés par les réformes est le secteur éducatif, qui a subi de profondes transformations à l'image des changements économiques et sociaux du pays.

\section{Les réformes des années 1990-2000}

2 Les lois de l'éducation nationale de 1997, puis de 2005, introduisent progressivement de nouveaux principes dans le système éducatif. Elles mettent l'accent sur l'ouverture du système, le changement du mode de gestion et le développement des valeurs civiques et démocratiques. La loi de 1997 fixe comme objectifs de «revoir les curricula et les contenus éducatifs à tous les niveaux d'enseignement; de renforcer les capacités et les initiatives individuelles des établissements en leur donnant plus de liberté et d'autonomie, d'encourager l'introduction des méthodes interactives d'enseignement et de renforcer le système d'information dans le domaine de l'éducation ». La loi de 2005 rappelle la nécessité «d'ouvrir le système éducatif à toutes les catégories de la population » et « de créer des conditions nécessaires pour promouvoir le développement de la conscience civique basée sur les valeurs libérales et démocratiques, avec le respect des valeurs culturelles nationales ».

3 Ainsi le modèle soviétique, marqué par une forte centralisation, est-il progressivement remplacé par un nouveau système de gestion décentralisée. Les établissements 
bénéficient dorénavant d'une plus grande autonomie, afin de mieux adapter leurs formations aux besoins des élèves.

Durant cette période, le ministère connaît de nombreuses réorganisations. À partir du milieu des années 2000, une partie de ses attributions est transférée à des agences externes (indépendantes du ministère en ce qui concerne le contenu de leur travail). Celles-ci sont en charge de questions telles que la mise en place du baccalauréat, l'évaluation de la qualité des programmes, etc. Les rectorats se voient accorder un nouveau rôle et deviennent des centres régionaux de ressources éducatives. Ceux-ci, contrairement au passé, ne contrôlent plus mais viennent en appui des établissements au niveau local pour de nombreuses questions (l'élaboration de la composante régionale dans les curricula, le renforcement des capacités administratives et financières des établissements, la mise en place des formations spécifiques des enseignants...).

Cependant, malgré une forte volonté de changement au niveau politique, la mise en pratique des nouveaux dispositifs s'avère difficile. De nombreux obstacles, liés d'une part à l'état économique et social du pays (manque de financements, faible niveau des salaires des enseignants et corruption) et d'autre part à la résistance naturelle du système au changement, empêchent la bonne réussite des réformes. En effet, le passage à la gestion décentralisée, impliquant une plus grande responsabilité de tous les acteurs du système, représente un défi important. On constate que les établissements peinent à se saisir pleinement de leurs nouveaux droits ou, au contraire, en abusent. D'autre part, les autorités centrales ne parviennent pas à établir un dialogue ouvert et constructif avec ces derniers. Ils ont encore tendance à employer des mesures autoritaires fortes pour tenter de réguler le système.

6 Une autre réforme significative dans le système éducatif géorgien est l'apparition, au début des années quatre-vingt-dix, du secteur privé. Cette ouverture a entraîné une forte croissance du nombre d'établissements à tous les niveaux d'enseignement et plus particulièrement au niveau de l'enseignement supérieur. La libéralisation a finalement été jugée excessive et l'intervention publique est devenue nécessaire afin de protéger le système des établissements frauduleux ou des formations de mauvaise qualité. Le travail d'accréditation des établissements d'enseignement supérieur conduit par le Centre national d'accréditation, entre 2005 et 2008, a permis en partie de remédier à ce problème 2 .

\section{Organisation et gestion du système éducatif}

7 En 2006, quatre agences externes au ministère ont été crées: le Centre national de programmes et d'évaluation des acquis, le Centre national des examens, le Centre national d'accréditation éducative et le Centre de développement professionnel des enseignants.

8 Le Centre national de programmes et d'évaluation est en charge du développement des programmes, de l'élaboration des systèmes d'évaluation, de la mise en conformité des manuels scolaires avec les standards éducatifs. Un des objectifs clés de ce centre consiste à rénover les programmes scolaires en y introduisant les méthodes d'apprentissage interactif et à remplacer les anciennes méthodes basées uniquement sur l'acquisition de savoirs factuels. 
9 L'introduction de l'examen national à la fin de l'enseignement obligatoire (équivalent du baccalauréat) occupe une place importante dans les réformes éducative récentes. Cet examen, mis en place pour la première fois en 2005-2006 par le Centre national des examens vise, entre autres, à combattre la corruption à l'entrée dans l'enseignement supérieur. En 2005-2006, la mise en œuvre de l'examen a été surveillée par Transparency International, qui a jugé la procédure conforme aux normes internationales. D’après une étude conduite par Transparency International auprès des étudiants et leurs parents, ce dispositif a bel et bien réussi à réduire la corruption si répandue dans l'administration universitaire.

10 Outre le suivi et la mise en place de l'examen du baccalauréat, le Centre gère également la répartition des places pour l'accès à l'enseignement supérieur. Il définit le nombre de places que les établissements peuvent proposer chaque année aux nouveaux étudiants et répartit les bacheliers dans différents établissements en fonction de leurs vœux. Ce nouveau système a entièrement remplacé les anciennes procédures par lesquelles chaque université gérait elle-même ses inscriptions à partir des résultats des concours d'entrée, qui étaient mis en place et élaborés par elle.

11 Le Centre national d'accréditation assure le contrôle de la qualité des programmes à tous les niveaux d'enseignement. Ce centre s'est vu confier une mission audacieuse : conduire l'évaluation de tous les établissements d'enseignement supérieur du pays. Suite à cette évaluation, le nombre d'établissements a été divisé presque par cinq, passant de 227 à 43 entre 2004 et 2007. Les deux dernières années, le nombre d'établissements accrédités a augmenté et compte aujourd'hui 62 institutions.

Le Centre de développement professionnel des enseignants a pour objectif d'élaborer les standards professionnels pour les métiers d'enseignement, les conditions de formation et de certification des enseignants. Il gère également les offres de formation continue.

13 Au niveau local, l'accent a été mis sur le renforcement des capacités de gestion des établissements. La loi de 2005 rend obligatoire la création, dans chaque établissement scolaire, d'un conseil d'administration, un organe de pilotage qui réunit les représentants de différentes instances (les municipalités, le ministère, les enseignants, les parents d'élèves, et les élèves). Le conseil définit les aspects majeurs de la vie de l'établissement: il élit le proviseur, établit le budget et vérifie sa mise en œuvre, décide du choix des manuels et des méthodes d'enseignement. Dans les établissements d'enseignement supérieur, un conseil administratif et un conseil académique assurent le pilotage et la mise en conformité avec les règles nationales, tous les deux avec une importante participation étudiante.

\section{Financements}

14 Au début des années 1990, on assiste à une forte chute des financements publics dans le secteur éducatif, qui ne représentent plus que $2 \%$ du PIB. L'État ouvre le système au financement privé (les établissements sont autorisés à chercher des sources de financement complémentaires et notamment à introduire les frais d'inscription), mais cette mesure ne s'avère pas suffisante et le manque de financements se fait ressentir de plus en plus fortement. Cette tendance s'inverse dans le milieu des années 2000, marquées par l'augmentation des financements publics ${ }^{3}$ et par une modification des principes de financement. 
15 Les établissements scolaires ne sont désormais plus sous la tutelle des collectivités territoriales. Leur budget est directement financé par l'État, avec des montants de subventions qui varient en fonction du nombre d'élèves inscrits et du type d'établissement (rural, urbain ou dans les zones géographiques éloignées).

Ce financement, dit par «vouchers », ne couvre que les dépenses courantes, les dépenses en capital étant financées dans le cadre de deux programmes ciblés : le Programme de Jacob Gogebashvili (construction et rénovation des bâtiments et des équipements scolaires) et le Programme "Saut de chevreuil $»^{4}$ (qui porte sur l'introduction des nouvelles technologies). Depuis 2005, année de lancement des deux programmes, 32 nouvelles écoles ont été construites et plus de 600 établissements ont été rénovées, plus de 26000 ordinateurs ont été installés. Le nombre d'élèves par ordinateur a été divisé par dix, passant à vingt élèves par ordinateur. En 2008, les deux types de financement représentaient respectivement 25,3 millions de laris (12,7 millions d'euros) et 12,3 millions de laris (6,2 millions d'euros). À titre de comparaison, pour cette même année, le montant total des dépenses du ministère de l'éducation pour l'enseignement général s'élevait à 268,6 millions de laris (134,2 millions d'euros).

17 Le même principe de financement s'applique au niveau de l'enseignement supérieur, où les subventions aux établissements sont versées sous forme de bourses qui couvrent les frais d'inscriptions des étudiants. En 2008, le montant de bourses versées aux établissements a atteint 21 millions de laris (10,5 millions d'euros). Le montant total des dépenses à destination de l'enseignement supérieur a atteint, en 2008, 48 millions de laris (24 millions d'euros). L'ensemble des dépenses à tous les niveaux d'enseignement s'élève à 316 millions de laris (158 millions d'euros), ce que représente 2,4\% du PIB.

18 Malgré les augmentations des dépenses publiques dans le secteur éducatif ces dernières années, ces efforts restent insuffisants. Actuellement, nombreuses sont encore les écoles avec des bâtiments délabrés et des salles de classe vétustes. Le manque de nouveaux matériels pédagogiques, les salaires faibles des enseignants et du personnel scolaire font partie des problèmes les plus importants à résoudre à l'avenir.

\section{Chiffres clés et la structure du système éducatif}

19 La durée de l'enseignement général est de douze ans. L'entrée à l'école primaire s'effectue à 6 ans, les études au collège débutent à 12 ans et le lycée à 15 ans. Au moment des réformes, la durée de l'enseignement primaire a augmenté de deux ans. En 2008, 2541 établissements d'enseignement général accueillaient 643000 élèves. Dans l'enseignement supérieur, on compte 94000 étudiants inscrits dans 62 établissements 5 .

20 Le nombre d'enseignants au niveau de l'enseignement général s'élève à 77011 . Plus des deux tiers sont titulaires d'un diplôme national de niveau mastère. La formation initiale des instituteurs, des professeurs des collèges et des lycées est délivrée par des instituts de formation ou via d'autres cursus universitaires.

21 Après le collège, les étudiants peuvent choisir de poursuivre des études professionnelles courtes qui débouchent sur une sortie professionnelle rapide. Une formation professionnelle longue est ouverte aux étudiants ayant fini l'enseignement obligatoire et titulaires d'un baccalauréat professionnel. De nombreux rapports soulignent la situation particulièrement difficile dans le domaine de l'enseignement professionnel. Le faible financement de l'État, une forte dégradation des équipements et un manque 
d'enseignants qualifiés sont pointés comme les principaux problèmes dans ce secteur. Un des aspects particulièrement sensibles est l'inadéquation entre les demandes du marché du travail et les formations proposées. Les réformes de l'enseignement professionnel font partie des priorités actuelles dans le domaine de l'éducation. Des études et des projets nationaux et internationaux se multiplient sur ce sujet afin de préparer les futures réformes ${ }^{6}$.

\section{Programmes scolaires et méthodes d'enseignement}

Dans le cadre des réformes des années 1990, la rénovation des méthodes et des orientations pédagogiques est devenue un élément important. Si, dans le système soviétique, l'accent était mis sur l'acquisition des savoirs factuels, les nouvelles méthodes d'enseignement visent à développer chez l'enfant l'esprit critique et une autonomie dans la capacité de réflexion.

L'enseignement de la langue nationale est une matière importante dans le nouveau curriculum, son apprentissage est obligatoire pour tous les élèves, avec un nombre d'heures plus important. Cependant, le géorgien peut également être enseigné comme seconde langue. La loi de 2005 autorise l'enseignement en langue minoritaire dans certaines zones géographiques du pays mais l'apprentissage du géorgien reste obligatoire dans toutes les écoles. Dans près de 500 écoles, l'enseignement se fait dans des langues minoritaires (dans 150 écoles en russe, 150 en arménien et 150 en azéri). Dans ces écoles, l'enseignement de la langue géorgienne en tant que seconde langue est obligatoire dès l'école primaire.

Parmi les autres matières obligatoires, on trouve également les mathématiques, les sciences (biologie, chimie et physiques), les sciences sociales (histoire, économie), les langues étrangères (russe ${ }^{7}$, anglais, allemand, français, ukrainien, espagnol, italien, turc, japonais et arabe), les arts et le travail manuel, la musique, les nouvelles technologies ou encore la culture physique. Au collègue et au lycée sont également enseignées l'histoire de la Géorgie et son organisation administrative et politique. À partir du lycée, l'apprentissage des deux langues étrangères est obligatoire.

Le redoublement est rare et la répartition des élèves par niveau n'est pas pratiquée. Selon la loi, la taille des classes ne peut pas excéder trente élèves mais en réalité, le nombre d'élèves par classe varie considérablement selon les zones. La différence entre les écoles urbaines (dans certains cas jusqu'à quarante élèves) et les écoles rurales (où les élèves de différents âges sont souvent regroupés dans la même classe pour éviter la fermeture d'écoles) est particulièrement prononcée.

\section{L'enseignement supérieur}

Les réformes dans l'enseignement supérieur ont été marquées par l'introduction du Processus de Bologne. L'adhésion au Processus a été, pour la Géorgie, à la fois un instrument politique et un outil pratique pour rénover le système.

Le changement majeur concerne l'introduction des diplômes de deux niveaux (licence et mastère) pour remplacer l'ancien système avec un seul niveau de diplôme possible (le diplôme de "spécialiste »). Ce diplôme était délivré par la majorité des établissements d'enseignement supérieur après l'achèvement des cinq années d'études supérieures. 

quatre ans à introduire avec succès toutes ses composantes. Aujourd'hui, les formations en deux niveaux (licence, mastère) sont dispensées dans tous les établissements d'enseignement supérieur, les crédits ECTS et le Supplément au diplôme sont délivrés pratiquement partout, les procédures d'assurance qualité ont été mises en place par le Centre national d'accréditation, qui a initié le travail d'évaluation et de pilotage par les résultats.

Cependant, malgré les résultats et les indicateurs officiels plutôt favorables, il faudra encore du temps pour que les réformes introduites soient entièrement assimilées par ces différents acteurs. En effet, certains hauts responsables connaissent encore des difficultés dans la compréhension des mécanismes du Processus de Bologne et de ses principaux objectifs. La mobilité académique, bien qu'étant fortement encouragée au niveau politique, rencontre de nombreux obstacles lors de sa mise en pratique.

Face à ces objectifs ambitieux, de nombreux défis restent encore à relever. Ces défis consistent tout d'abord à renforcer la démocratisation et la décentralisation du système éducatif. Une meilleure intégration des personnes handicapées, le renforcement de l'éducation adaptée pour les minorités ethniques et le développement de l'éducation interculturelle sont également visés par les politiques publiques actuelles.

Quelques données sur la Géorgie (2008-2009)

\begin{tabular}{|l|l|}
\hline Superficie & $69700 \mathrm{~km}^{2}$ \\
\hline Population & 4,6 millions \\
\hline $\begin{array}{l}\text { Part de la population étrangère (azéri, arménien, russe, } \\
\text { autres) }\end{array}$ & $17,2 \%$ \\
\hline Religions principales & chrétiens \\
\hline & orthodoxes (84\%) \\
\hline & musulmans (9\%) \\
\hline & catholiques (1\%) \\
\hline Langues majeures & géorgien (71 \%) \\
\hline & russe (9 \%) \\
\hline & arménien (7 \%) \\
\hline Femmes & azéri (6 \%) \\
\hline Hommes & \\
\hline & 73,4 ans \\
\hline & 80,4 ans \\
\hline & \\
\hline &
\end{tabular}




\begin{tabular}{|c|c|}
\hline PIB par habitant & $\$ 4700$ \\
\hline Croissance annuel du PIB & $\begin{array}{l}2,4 \% \text { en } 2008 ; 12,4 \% \text { en } 2007 ; 9,4 \% \text { en } \\
2006\end{array}$ \\
\hline Taux de chômage & $13,6 \%$ \\
\hline \multicolumn{2}{|l|}{ Taux d'alphabétisation } \\
\hline Hommes & $100 \%$ \\
\hline Femmes & $99 \%$ \\
\hline \multicolumn{2}{|c|}{ Taux de scolarisation par rapport à la classe d'âge (2006) } \\
\hline Maternelle & $55 \%$ \\
\hline Primaire & $96 \%$ \\
\hline Secondaire & $85 \%$ \\
\hline Supérieur & $38 \%$ \\
\hline
\end{tabular}

\section{BIBLIOGRAPHIE}

MIMINOSHVILI M., JANASHIA S. (2007) : « Le système éducatif en Géorgie », in Encyclopédie Education PIRLS. A Guide to Reading Education in the Forty PIRLS 2006 Countries, Kennedy, A.M., Mullis, I.V.S., Martin, M.O., Trong, K.L. (Ed), Chestnut Hill, MA: TIMSS \& PIRLS International Study Center, Boston College.

UNESCO BUREAU INTERNATIONAL DE L'ÉDUCATION (2003) : « Le système éducatif géorgien », in Données mondiales de l'éducation, http://www.ibe.unesco.org/fr/dans-le-monde/europe-etamerique-du-nord/georgie/profile-of-education.html .

MINISTÈRE DE L'ÉDUCATION ET DE LA RECHERCHE (2007) : Consolidated Education Action And Strategy Plan 2007-2011, Ministère de l'Éducation et de la Recherche, Tbilissi.

\section{NOTES}

1. La Géorgie est un pays de 4,6 millions d'habitants qui s'étend sur environ $70000 \mathrm{~km}^{2}$ et se situe dans la région du Caucase, au croisement de l'Europe et l'Asie. Elle est bordée par la mer Noire sur la côte Ouest et partage sa frontière avec la Turquie et l'Arménie au sud, à l'est avec l'Azerbaïdjan et au nord avec la Russie. 
2. Aujourd'hui, malgré la fermeture de nombreux établissements privés d'enseignement supérieur, le secteur privé accueille encore $60 \%$ des effectifs. Dans l'enseignement général, ce pourcentage est de $7 \%$.

3. Augmentation de $35 \%$ au niveau de l'enseignement général (de 199,7 millions de laris en 2005 à 268,6 laris en 2008) et de $41 \%$ au niveau de l'enseignement supérieur (de 34,3 à 48 millions de laris pour la même période). Note : 1 laris $=0,50$ euro. Source : statistiques du ministère géorgien de l'éducation.

4. Ce programme a été développé à l'instar d'un programme mis en place en Estonie, «Saut de tigre ".

5. Ce chiffre correspond au nombre des établissements accrédités par l'État.

6. En 2008 , le ministère de l'éducation et des sciences géorgien a préparé un rapport qui fait un état des lieux dans le domaine de l'enseignement professionnel et propose de futures pistes d'action.

7. Le russe est enseigné dès la troisième classe à l'école primaire.

\section{INDEX}

Mots-clés : système éducatif

Index géographique : Géorgie

\section{AUTEURS}

\section{LISA BYDANOVA}

Chargée de programmes au Centre international d'études pédagogiques (CIEP). Titulaire d'un doctorat en économie de l'éducation de l'IREDU (Institut de recherche sur la sociologie et l'économie de l'éducation, Université de Bourgogne), elle a participé à des études et à des recherches sur les comparaisons des systèmes éducatifs auprès de la Banque mondiale, de l'Agence française de développement et de l'Institut de l'éducation de Londres.

\section{LELA MAISURADZE}

Lela Maisuradze a été responsable du département de l'harmonisation et de l'intégration internationale de l'enseignement supérieur au ministère de l'éducation et de la recherche de Géorgie. Elle travaille actuellement au ministère en tant qu'expert principal au Département des relations internationales et de l'intégration à l'OTAN et à l'Union européenne. 